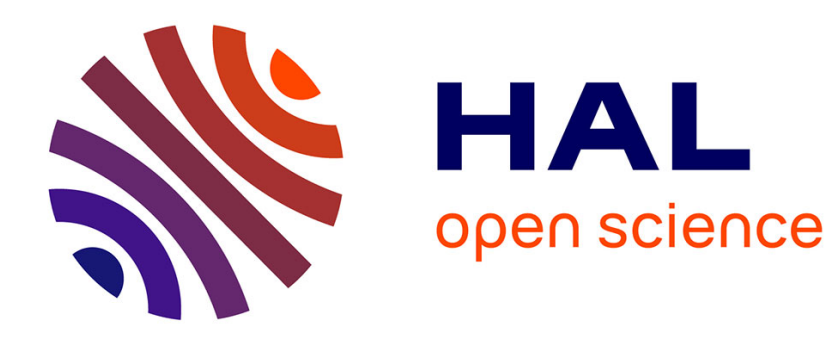

\title{
Exact marginals and normalizing constant for Gibbs distributions
}

\author{
Cécile Hardouin, Xavier Guyon
}

\section{To cite this version:}

Cécile Hardouin, Xavier Guyon. Exact marginals and normalizing constant for Gibbs distributions. C. R. Acad. Sci. Paris, Ser. I., 2010, 348, pp.199-201. hal-00795132

\section{HAL Id: hal-00795132 \\ https://hal.science/hal-00795132}

Submitted on 27 Feb 2013

HAL is a multi-disciplinary open access archive for the deposit and dissemination of scientific research documents, whether they are published or not. The documents may come from teaching and research institutions in France or abroad, or from public or private research centers.
L'archive ouverte pluridisciplinaire HAL, est destinée au dépôt et à la diffusion de documents scientifiques de niveau recherche, publiés ou non, émanant des établissements d'enseignement et de recherche français ou étrangers, des laboratoires publics ou privés. 
Statistics

\title{
Exact marginals and normalizing constant for Gibbs distributions
}

\author{
C. Hardouin ${ }^{\mathrm{a}, 1}, \mathrm{X}$. Guyon ${ }^{\mathrm{a}}$ \\ ${ }^{\text {a } C E S / S A M O S-M A T I S S E / U n i v e r s i t e ́ ~ d e ~ P a r i s ~} 1$
}

\begin{abstract}
We present a recursive algorithm for the calculation of the marginal of a Gibbs distribution $\pi$. A direct consequence is the calculation of the normalizing constant of $\pi$.
\end{abstract}

Résumé

Récurrences et constante de normalisation pour des modèles de Gibbs. Nous proposons dans ce travail une récurrence sur les lois marginales d'une distribution de Gibbs $\pi$. Une conséquence directe est le calcul exact de la constante de normalisation de $\pi$.

\section{Introduction}

Usually, obtaining the marginals and/or the normalizing constant $C$ of a discrete probability distribution $\pi$ involves high dimensional summation : for example, for the binary Ising model on a simple grid $10 \times 10$, the calculation of $C$ involves $2^{100}$ terms. One way to prevent this problem is to change distribution of interest for an alternative as, for example in spatial statistics, replacing the likelihood for the conditional pseudo likelihood ([1]). Another solution consists of estimating the normalizing constant; see for example Pettitt \& al ([8]) and Moeller \& al ([7]) for efficient Monte Carlo methods, Bartolucci and Besag ([2]) for a recursive algorithm computing the exact likelihood of a Markov random field, Reeves and Pettitt ([9]) for an efficient computation of the normalizing constant for a factorisable model.

We present specific results for a Gibbs distribution $\pi$. We derive results of Khaled $([5,6])$ who gives an original linear recursion on the marginals of $\pi$, the law of $Z=\left(Z_{1}, Z_{2}, \cdots, Z_{T}\right) \in E^{T}$; this result eases the calculation of $\pi$ 's normalizing constant. We generalize Khaled results noticing that if $\pi$ is a Gibbs

\footnotetext{
Email addresses: Cecile.Hardouin@univ-paris1.fr (C. Hardouin), guyon@univ-paris1.fr (X. Guyon).

1 Adresse de correspondance : C. Hardouin, SAMOS-MATISSE/Université de Paris 1, 90 rue de Tolbiac, 75634 Paris Cedex 13
} 
distribution on $\mathcal{T}=\{1,2, \cdots, T\}$, then $\pi$ is a Markov field on $\mathcal{T}$, so it is easy to manipulate its conditional distributions that are the basic tools of our forward recursions.

\section{Markov representations of a Gibbs field}

Let $T>0$ be a fix positive integer, $E=\left\{e_{1}, e_{2}, \cdots, e_{N}\right\}$ a finite state space, $Z=\left(Z_{1}, Z_{2}, \cdots, Z_{T}\right) \in E^{T}$ a temporal sequence with distribution $\pi$. Let us denote $z(t)=\left(z_{1}, z_{2}, \cdots, z_{t}\right)$. We assume that $\pi$ is a Gibbs distribution with energy and potentials:

$$
\begin{aligned}
& \pi(z(T))=C \exp U_{T}(z(T)) \text { with } C^{-1}=\sum_{z(T) \in E^{T}} \exp U_{T}(z(T)) \text { where } \\
& U_{t}(z(t))=\sum_{s=1, t} \theta_{s}\left(z_{s}\right)+\sum_{s=2, t} \Psi_{s}\left(z_{s-1}, z_{s}\right) \text { for } 2 \leq t \leq T, \text { and } U_{1}\left(z_{1}\right)=\theta_{1}\left(z_{1}\right) .
\end{aligned}
$$

So, $\pi$ is a bilateral 2 nearest neighbours Markov field $([4,3])$

$$
\pi\left(z_{t} \mid z_{s}, 1 \leq s \leq T \text { and } s \neq t\right)=\pi\left(z_{t} \mid z_{t-1}, z_{t+1}\right)
$$

but $Z$ is also a Markov chain :

$$
\pi\left(z_{t} \mid z_{s}, s \leq t-1\right)=\pi\left(z_{t} \mid z_{t-1}\right) \text { if } 1<t \leq T .
$$

An important difference appears between formulas (3) and (2): indeed, (2) is computationnally feasible, when (3) is not.

\section{Recursion over marginal distributions}

\subsection{Future-conditional contribution $\Gamma_{t}(z(t))$}

For $t \leq T-1$, the distribution $\pi\left(z_{1}, z_{2}, \cdots, z_{t} \mid z_{t+1}, z_{t+2}, \cdots, z_{T}\right)$ conditionally to the future, depends only on $z_{t+1}$ :

$$
\pi\left(z_{1}, z_{2}, \cdots, z_{t} \mid z_{t+1}, z_{t+2}, \cdots, z_{T}\right)=\frac{\pi\left(z_{1}, z_{2}, \cdots, z_{T}\right)}{\sum_{u_{1}^{t} \in E^{t}} \pi\left(u_{1}^{t}, z_{t+1}, . . z_{T}\right)}=\pi\left(z_{1}, z_{2}, \cdots, z_{t} \mid z_{t+1}\right) .
$$

We can also write $\pi\left(z_{1}, z_{2}, \cdots, z_{t} \mid z_{t+1}\right)=C_{t}\left(z_{t+1}\right) \exp U_{t}^{*}\left(z_{1}, z_{2}, \cdots, z_{t} ; z_{t+1}\right)$ where $U_{t}^{*}$ is the futureconditional energ :

$$
U_{t}^{*}\left(z_{1}, z_{2}, \cdots, z_{t} ; z_{t+1}\right)=U_{t}\left(z_{1}, z_{2}, \cdots, z_{t}\right)+\Psi_{t+1}\left(z_{t}, z_{t+1}\right)
$$

and $C_{t+1}\left(z_{t+1}\right)^{-1}=\sum_{u_{1}^{t} \in E^{t}} \exp \left\{U_{t}^{*}\left(u_{1}, \ldots, u_{t} ; z_{t+1}\right)\right\}$. Then, for $i=1, N$ :

$$
\pi\left(z_{1}, z_{2}, \cdots, z_{t} \mid z_{t+1}=e_{i}\right)=C_{t}\left(e_{i}\right) \gamma_{t}\left(z_{1}, z_{2}, \cdots, z_{t} ; e_{i}\right) \text { where } \gamma_{t}\left(z(t) ; e_{i}\right)=\exp U_{t}^{*}\left(z(t) ; e_{i}\right) .
$$

With the convention $\Psi_{T+1} \equiv 0$, we define for $t \leq T$, the vector $\Gamma_{t}(z(t)) \in \mathbb{R}^{N}$ of the future-conditional contributions as

$$
\left(\Gamma_{t}(z(t))\right)_{i}=\gamma_{t}\left(z(t) ; e_{i}\right), 1 \leq i \leq N
$$

and the recursion matrix $A_{t}$ by

$$
A_{t}(i, j)=\exp \left\{\theta_{t}\left(e_{j}\right)+\Psi_{t+1}\left(e_{j}, e_{i}\right)\right\}, i, j=1, N .
$$

Then we get the following fundamental recurrence. 
Proposition 3.1 For all $2 \leq t \leq T, z(t)=\left(z_{1}, z_{2}, \cdots, z_{t}\right) \in E^{t}$ and $e_{i} \in E$, we have:

$$
\gamma_{t}\left(z(t-1), e_{j} ; e_{i}\right)=A_{t}(i, j) \times \gamma_{t-1}\left(z(t-1) ; e_{j}\right),
$$

and

$$
\sum_{z_{t} \in E} \Gamma_{t}\left(z(t-1), z_{t}\right)=A_{t} \Gamma_{t-1}(z(t-1))
$$

\subsection{Forward recursions on marginals and normalization constant}

Let us define the following $1 \times N$ row vectors : $E_{1}=B_{T}=(1,0, \cdots, 0)$, and the $\left(B_{t}\right)_{t=T, 2}$ defined by the forward recursion $B_{t-1}=B_{t} A_{t}$ if $t \leq T$; we also denote $K_{1}=\sum_{z_{1} \in E} \Gamma_{1}\left(z_{1}\right) \in \mathbb{R}^{N}$. We give below the main result of this work.

Proposition 3.2 Marginal distributions $\pi_{t}$ and calculation of the normalization constant $C$.

(1) For $1 \leq t \leq T$ :

$$
\pi_{t}(z(t))=C \times B_{t} \Gamma_{t}(z(t)) .
$$

(2) The normalization constant $C$ of the joint distribution $\pi$ verifies:

$$
C^{-1}=E_{1} A_{T} A_{T-1} \cdots A_{2} K_{1} .
$$

The formula (9) reduces to $C^{-1}=E_{1} A_{T} A^{T-2} K_{1}$ for time invariant potentials.

As a basic example, let us consider $E=\{0,1\}, \theta_{t}\left(z_{t}\right)=\alpha z_{t}$, and $\Psi_{t+1}\left(z_{t}, z_{t+1}\right)=\beta z_{t} z_{t+1}$; the analytic expressions of $A, K_{1}$ are trivially derived. We computed $C^{-1}=E_{1} A_{T} A^{T-2} K_{1}$ for increasing values of $T$; the computing time is always negligible for $T \leq 700$, whereas computing $C^{-1}$ by direct summation needs 750 seconds for $T=20,6$ hours for $T=25$, and the method becoming ineffectual for $T>25$.

\section{Extensions to general Gibbs fields}

There are various generalisations of the preceeding results.

\subsection{Temporal Gibbs model}

Let us give the following example as an illustration to possible extensions. Coming back to the previous model (1), we add the interaction potentials $\Psi_{2, s}\left(z_{s-2}, z_{s}\right)$. Then $\pi$ is a 4 nearest neighbours Markov field but also a Markov chain of order 2. Conditionally to the future, we get

$$
\begin{gathered}
\pi\left(z_{1}, z_{2}, \cdots, z_{t} \mid z_{t+1}, z_{t+2}, \cdots, z_{T}\right)=\pi\left(z(t) \mid z_{t+1}, z_{t+2}\right)=C_{t}\left(z_{t+1}, z_{t+2}\right) \exp U_{t}^{*}\left(z(t) ; z_{t+1}, z_{t+2}\right), \text { with } \\
U_{t}^{*}\left(z(t) ; z_{t+1}, z_{t+2}\right)=U_{t}(z(t))+\Psi_{1, t+1}\left(z_{t}, z_{t+1}\right)+\Psi_{2, t+1}\left(z_{t-1}, z_{t+1}\right)+\Psi_{2, t+2}\left(z_{t}, z_{t+2}\right),
\end{gathered}
$$

Then, for $a, b$ and $c \in E, U_{t}^{*}(z(t-1), a ;(b, c))=U_{t-1}^{*}(z(t-1) ;(a, b))+\theta_{t}(a)+\Psi_{1, t+1}(a, b)+\Psi_{2, t+2}(a, c)$; analogously to the previous example, we define the future-conditional contributions and the $N^{2} \times N^{2}$ matrices $A_{t}$ by

$$
\begin{aligned}
\gamma_{t}\left(z(t) ;\left(z_{t+1}, z_{t+2}\right)\right) & =\exp U_{t}^{*}\left(z(t) ;\left(z_{t+1}, z_{t+2}\right)\right. \\
A_{t}((i, j),(k, i)) & =\exp \left\{\theta_{t}\left(e_{k}\right)+\Psi_{1, t+1}\left(e_{k}, e_{i}\right)+\Psi_{2, t+2}\left(e_{k}, e_{j}\right)\right\}
\end{aligned}
$$


Similarly as 3.1, we get the following recursion:

$$
\gamma_{t}\left(z(t-1), e_{k} ;\left(e_{i}, e_{j}\right)\right)=A_{t}((i, j),(k, i)) \times \gamma_{t-1}\left(z(t-1) ;\left(e_{k}, e_{i}\right)\right)
$$

We thus obtain a recurrence (7) on the contributions $\Gamma_{t}(z(t))$ and analogous results as (8) and (9) for the bivariate Markov chain $\left(Z_{t-1}, Z_{t}\right), t=1, T$.

\subsection{Spatial Gibbs fields}

For $t \in \mathcal{T}=\{1,2, \ldots T\}$, let us consider $Z_{t}=\left(Z_{(t, i)}, i \in \mathcal{I}\right)$, where $\mathcal{I}=\{1,2, \cdots, m\}, Z_{(t, i)} \in F$. Then $Z=\left(Z_{s}, s=(t, i) \in \mathcal{S}\right)$ is a spatial field on $\mathcal{S}=\mathcal{T} \times \mathcal{I}$. We note again $z_{t}=\left(z_{(t, i)}, i \in \mathcal{I}\right), z(t)=\left(z_{1}, . ., z_{t}\right)$, $z=z(T)$ and we suppose that the distribution $\pi$ of $Z$ is a Gibbs distribution with translation invariant potentials $\Phi_{A_{k}}(\bullet), k=1, K$ associated to a family of subsets $\left\{A_{k}, k=1, K\right\}$ of $\mathcal{S}$. For $A \subseteq \mathcal{S}$, let us define $H(A)=\sup \{|u-v|, \exists(u, i)$ and $(v, j) \in A\}$, and $H=\sup \left\{H\left(A_{k}\right), k=1, K\right\}$. With this notation, we write the Gibbs-energy

$$
U(z)=\sum_{h=0}^{H} \sum_{t=h+1}^{T} \Psi\left(z_{t-h}, \cdots, z_{t}\right) \text { with } \Psi\left(z_{t-h}, \cdots, z_{t}\right)=\sum_{k: H\left(A_{k}\right)=h} \sum_{s \in S_{t}(k)} \Phi_{A_{k}+s}(z)
$$

where $S_{t}(k)=\left\{s=(u, i): A_{k}+s \subseteq \mathcal{S}\right.$ and $\left.t-H\left(A_{k}\right) \leq u \leq t\right\}$. Then $\left(Z_{t}\right)$ is a Markov process of order $H$ and $Y_{t}=\left(Z_{t-H}, Z_{t-H+1}, \cdots Z_{t}\right), t>H$ a Markov chain on $E^{H}$ for which we get the results (8) and (9).

We applied the result to the calculation of the normalization constant for an Ising model. For $m=10$ and $T=100$, the computing time is less than 20 seconds.

\section{References}

[1] J. Besag, 1974. Spatial interactions and the statistical analysis of lattice systems. J. Roy. Statist. Soc. B 148, 1-36

[2] F. Bartolucci, J. Besag, 2002. A recursive algorithm for Markov random fields. Biometrika 89 3, 724-730

[3] X. Guyon, 1995. Random Fields on a Network: Modeling, Statistics, and Applications. Springer-Verlag, New York

[4] R. Kindermann, J.L. Snell, 1980. ), Markov random fields and their applications, Contemp. Maths.

[5] M. Khaled, 2008. A multivariate generalization of a Markov switching model. working paper C.E.S. Université Paris 1, http://mkhaled.chez-alice.fr/khaled.html

[6] M. Khaled, 2008. Estimation bayésienne de modèles espace-état non linéaires. Ph. D. Thesis, Université Paris 1, http://mkhaled.chez-alice.fr/khaled.html

[7] J. Moeller, A.N. Pettitt, R. Reeves, K.K. Berthelsen, 2006. An efficient Markov chain Monte Carlo method for distributions with intractable normalizing constants. Biometrika 93 2, 451-458.

[8] A.N. Pettitt, N. Friel, R. Reeves, 2003. fficient calculation of the normalizing constant of the autologistic and related models on the cylinder and lattice. J. Roy. Statist. Soc. B 65, part 1, 235-246

[9] R. Reeves,A.N. Pettitt, 2004. Efficient recursions for general factorisable models. Biometrika 91 3, 751-757. 